\section{Lipids: Evergreen autofluores- cent biomarkers for the liver functional profiling}

\author{
Anna Cleta Croce, Giovanni Bottiroli \\ Histochemistry and Cytometry Unit, \\ IGM-CNR, Biotechnology and Biology \\ Department, University of Pavia, Italy
}

\begin{abstract}
Depending on their chemical nature, lipids can be classified in two main categories: hydrophilic, greatly contributing to membrane composition and subcellular organelle compartmentalization, and hydrophobic, mostly triglycerides, greatly enrolled in the storage and production of energy. In both cases, some lipid molecules can be involved as signaling agents in the regulation of metabolism and protective or damaging pathways in responses to harmful stimuli. These events could affect in particular the liver, because of its central role in the chiefly present in the myelin sheath surrounding the axons of some nerve cells, and their histochemical detection allows to identify alterations in both the central and peripheral nervous system, for diagnostic purposes in neuropathology and experimental neurology. ${ }^{2}$ Lipids with hydrophilic or amphiphilic properties, such as glycerolphospholipids and sterols can form vesicles in the aqueous medium and greatly participate to the composition of plasmalemma and the membranes of subcellular organelles. Lipid droplets are the main intracellular storage sites of fatty acids, in the form of hydrophobic, neutral triglycerides.
\end{abstract} maintenance of lipid homeostasis. Lipids have been demonstrated to fluoresce, contributing to the overall emission signal of the liver tissue along with other endogenous fluorophores, relatable to energy metabolism and oxidative events. The mere estimation of the fluorescing lipid fraction in parallel with the other endogenous fluorophores, and with the common biochemical and histochemical biomarkers of tissue injury has been exploited to investigate the liver morpho-functional conditions in experimental hepatology. More interestingly, the fluorescing lipid fraction is greatly relatable to free fatty acids such as arachidonic, linoleic and linolenic acid, which are deserving increasing attention as precursors of products involved in several and complex signaling pathways. On these bases, the ability of autofluorescence to detect directly arachidonic acid and its balance with other unsaturated fatty acids may be exploited in the diagnosis and follow-up of fatty livers, helping to improve the personalization of the metabolic/lipidomic profiling. This could also contribute to elucidate the role of the injuring factors in the choice of suitable donors, and in the set-up of preservation procedures in liver transplantation.

\section{Introduction}

Recently renewed notices on histochemical methodologies have reaffirmed the critical role of fixation and of different staining procedures for the in situ discrimination of the various kinds of lipids. ${ }^{1-3}$ The chemical nature and localization of lipids can be evidenced by means of various procedures, to be performed on fixed or frozen unfixed samples, or even in living conditions, depending on the balance in the need to preserve the structure and the biochemistry of cells and tissues, according to the morphological or metabolic targets aimed to be investigated. ${ }^{1,4}$ Sphingomyelins are

\section{Lipids in signal transduction and as diagnostic factors}

Within the several functional activities, beyond membrane compartmentalization and trafficking, energy storage and production, lipids can participate to signal transduction in the modulation of hormone actions, metabolism, and protective or damaging responses to hazardous stimuli. ${ }^{4,5}$ and references therein

A classical signaling strategy is based on the action of phospholipases. This is the case of phosphatidylinositol phosphorylation, with the production of various phosphoinositides as cell surface signaling agents for the regulation of various membrane functions, cytoskeletal organization, and nuclear events. ${ }^{6}$ Likewise, the lipid droplets which are ubiquitously present in eukaryotic organisms, are recognized as dynamic bioactive agents. The mere presence of lipid droplets has been for example investigated by Oil Red staining and image analysis, to estimate the changes in their amount in single cultured cell models during differentiation, ${ }^{7}$ or to develop reliable, standardized integrated optical density procedures to evaluate their size and distribution in healthy and pathological cell populations. ${ }^{8,9}$ Actually, the rising of severe metabolic and degenerative diseases, up to the cancer, are commonly considered to be
Correspondence: Dr. Anna Cleta Croce, IGMCNR, Sezione Istochimica e Citometria, Dipartimento di Biologia e Biotecnologie "L. Spallanzani", Universita degli Studi di Pavia, via A. Ferrata 9, 27100 Pavia, Italy.

Tel. +39.0382.986428 - Fax: +39.0382 .986430 . E-mail: croce@igm.cnr.it

Key words: Arachidonic acid; autofluorescence; endogenous fluorophores; liver; personalized diagnosis.

Conflict of interest: the authors report no conflict of interest.

Received for publication: 11 May 2017.

Accepted for publication: 25 May 2017.

This work is licensed under a Creative Commons Attribution-NonCommercial 4.0 International License (CC BY-NC 4.0).

CCopyright A.C. Croce and G. Bottiroli, 2017 Licensee PAGEPress, Italy

European Journal of Histochemistry 2017; 61:2808 doi:10.4081/ejh.2017.2808

greatly favored by the concurrent disruption of lipid metabolism and signaling actions of fatty acids, both mobilized from the lipid droplets or derived extracellularly from the diet, and their products. ${ }^{10-12}$ In this respect, it is worth to remind that the role of lipids in the development of metabolic diseases and cancer is also supported by studies based on magnetic resonance imaging and magnetic resonance spectroscopy, which have been compared to an in vivo histochemical technique able to identify both the chemical nature and the spatial distribution of lipids in different tissues and organs, including the liver. ${ }^{13}$, and references therein

Lipids can therefore be considered as important diagnostic factors.

\section{Lipids in hepatology}

The central role played by the liver in the storage and keeping on the systemic homeostasis of nutrients, including lipids, makes it particularly exposed to the consequences of an altered lipid metabolism, in particular in the presence of excess of lipids and oxidative stress. This is the case for Non Alcoholic Liver Disease (NAFLD): this pathology is the cause of heavy social and economical issues in the human population, because of its incidence (about $30 \%$ of the individuals in Western countries), the associated increased risk of progression to more severe disease, and the need to recruit fatty livers as marginal organs to enhance 
the availability of donors for liver transplantation. ${ }^{14-16}$

NAFLD is currently reported as a complex multisystemic disease, ${ }^{15-17}$ following the so-called 'two hits' hypothesis, according to which the lipid accumulation is the first 'hit' that requires a second 'hit'(commonly oxidative stress) for the progression of the disease. This concept is greatly sustained by the numerous studies comparing various rodent models of NAFLD and the pathophysiological features of the human disease, which directed a growing attention to the lipids. ${ }^{18}$ For example, fatty livers from nutritional rat models (e.g., the Methionine Choline deficient diet, MCDrats) can undergo a spontaneous activation of inflammation and an effective progression to fibrosis as compared to the genetic (fa/fa obese Zucker) rats, which, anyway, have lesser oxidative stress and are richer in triglycerides. ${ }^{18,}$ and references therein The microspectrofluorometric analyses of unfixed, unstained liver sections after Nile red fluorochromization have confirmed the prevailing presence of neutral lipids in both lean and obese Zucker rats, as compared to MCD and control Wistar rat. ${ }^{19}$

\section{Fluorescing lipids for the liver functional profiling}

Notably, some lipids can give rise to a native emission of fluorescence. This feature makes lipids to act as possible endogenous fluorophores, improving the diagnostic potential of autofluorescence. In fact, the overall autofluorescence emission properties depend on the presence, nature, amount and physico-chemical conditions of the endogenous biomolecules strictly related with the morpho-functional features of normal or altered cells and tissue. As a consequence, autofluorescence carries a comprehensive information on the physiological, normal or altered conditions of the biological substrate, and can be exploited for in situ, real time diagnostic purposes. ${ }^{20}$

A lower contribution of fluorescing lipids to the overall liver autofluorescence emission was detected in the lean Zucker rats as compared with the obese ones, in agreement with the results on the proportions of polyunsaturated fatty acids reported on the same models by Fukumori et al. ${ }^{19,21}$ Conversely, an autofluorescence investigations on the effects of acute starvation on the hepatic tissue of Wistar rats showed that the fraction of fluorescing lipids was poorly affected, in front of a significant increase in retinoids and lipid droplets in the starved rats, as proved by histochemistry. ${ }^{22}$ All together, these data indicate an engagement of each specific group of rats in keeping constant the fraction of the liver fluorescing lipids. Consistently, no great variations were shown by fluorescing lipids in livers submitted to a two hour normothermic reperfusion, following a previous organ isolation from MCD and lean control Wistar rats and preservation under cold storage or after mechanical perfusion at subnormothermic $\left(20^{\circ} \mathrm{C}\right)$ temperature (MP-20), except initial changes indicating a mobilization at the beginning of the reperfusion of MCD livers. ${ }^{23}$

The lipid fluorescing fraction has been mostly identified as arachidonic acid (AA) by comparative spectroscopic studies on the autofluorescence of hepatic tissue extracts and pure compounds. ${ }^{24}$ The AA dominance in the liver fluorescing lipids has been supported by additional studies on different single free fatty acids, demonstrating a much greater fluorescence yield of AA than linoleic and oleic acids. ${ }^{25}$ Therefore, the autofluorescence of AA can be proposed as a supportive diagnostic tool in experimental hepatology, as to both the mere balance between the free and immobilized fractions of lipids composing the membranes and influencing their fluidity, and the more complex and not yet fully clarified bioactive functions in mediating the responses to stress factors. ${ }^{26}$

\section{Fatty livers for organ transplan- tation: Lipids and the regulation of functional preservation}

Several studies have highlighted the importance of AA in the balance of hepatic injury and protection, with the modulation of cell functions, inflammation and apoptosis through the cascade of various intermediates, as well as the cross-talking with vasodilators and cytokines, as for example Nitric Oxide (NOx) and the Tumor Necrosis Factor-alfa (TNF-alfa). ${ }^{26-30}$ With particular reference to fatty livers as marginal donor organs for transplantation, the improvement in the knowledge on the responses of intermediate agents to injuring factors is critical for the set-up of personalized interventions to ameliorate preservation and the graft outcome. For example, the conditions of temperature and oxygenation applied in the organ preservation have been found to induce different responses form lean and obese Zucker rat liver models. The bile production, which reflects the integrity of biliary epithelial cells, and ATP, significant of the tissue energy content, indicated a better outcome of fatty livers under MP-20 conditions as compared with static and/or lower temperature conditions. In general fatty livers submitted to MP-20 showed a lower release of intratissutal enzymes (Lactate De-Hydrogenase, LDH; Aspartate Amino Transferase, AST), which even approached the levels of the lean livers submitted to the same procedures. ${ }^{31,32}$ and a lesser increase in NOx, TNF-alfa and early apoptosis. ${ }^{31,33}$ Histochemistry demonstrated that in comparison with both lean livers and preservation at lower temperature, the fatty livers submitted to MP-20 did not show significant changes in the number of apoptotic (i.e., caspase-3- and TUNEL-positive) hepatocytes, with a limited increase in the positivity for the M30 biomarker, specific for the caspase-cleaved fragment of cytokeratin 18. The hepatocyte death via apoptosis in fatty livers was reduced, in parallel with a recovery of glycogen stores, a decrease in the macrosteatosis patterns and a lesser presence of ROS production. All together these results indicate that, beyond the lipid composition already designated as a relevant cause of the greater susceptibility to the preservation temperature of lean as compared with fatty Zucker livers, ${ }^{21,31,32}$ the beneficial effects of MP-20 involve complex and interrelated metabolic and signaling factors. Interestingly, histochemistry showed also a prevalence of the apoptosis biomarkers, glycogen stores, and ROS production in the periportal, mid-zone areas, ascribed to the predominance of the oxidative energy metabolism in the periportal zone sustained by a much higher oxygen supply. ${ }^{34}$ In this regard, the importance of vascularization could also explain the interlobe differences in peroxidation of lipids and TNF-alpha detected after ischemia/ reperfusion of livers from Sprague Dawley, likely mediating the activation of tissue matrix metalloproteinases. ${ }^{35}$ These findings fit with the common views on the balance between various factors and mechanisms in the modulation of tissue injury following hepatic stress, including the regulation of blood circulation or inflammation, and the possible role of AA derived products in their activation. ${ }^{28}$

\section{Concluding remarks}

The heterogeneous liver functional and structural properties have been more recently reconsidered by focusing on the zonation in the lipid droplet accumulation and the remodeling in the AA mobilization, production of the pro-inflammatory signaling molecules, eicosanoids and associated oxida- 
tive damage in NAFLD and disease progression. ${ }^{36,37}$ In parallel, promising findings have been reported on some metabolites of polyunsaturated fatty acids, in particular the AA-derived eicosanoids, and their potential role as biomarkers of NAFLD, promoting their further investigation as adjunctive tools in the panel of modestly-invasive blood biomarkers in the diagnosis of fatty liver diseases. ${ }^{38}$ In this context, the ability of autofluorescence to detect the free fraction of AA not only in the liver tissue, but also in the crude serum, ${ }^{25,39}$ can be proposed as a promising additional biomarker in hepatology. Multiple possible applications can be proposed, from the support to elucidate the role of the injuring factors in the preselection and set-up of preservation of donor livers for transplantation, to the development of a relatively low-cost, time effective supportive diagnostic tool in the clinical practice, for a personalized metabolic/lipidomic profiling in the diagnosis of fatty livers. ${ }^{14,40}$

\section{References}

1. Carriel V, Campos F, AneirosFernández J, Kiernan JA. tissue fixation and processing for the histological identification of lipids. Methods Mol Biol 2017;1560:197-206

2. Carriel V, Campos A, Alaminos M, Raimondo S, Geuna S. Staining methods for normal and regenerative myelin in the nervous system. Methods Mol. Biol 2017;1560:207-18.

3. Rostron KA, Lawrence CL. Nile red staining of neutral lipids in yeast. Methods Mol Biol 2017;1560:219-29.

4. Bader CA, Shandala T, Carter EA, Ivask A, Guinan T, Hickey SM, et al. A molecular probe for the detection of polar lipids in live cells. PLoS One 2016;11:e0161557.

5. Wymann MP, Schneiter R. Lipid signalling in disease. Nat Rev Mol Cell Biol 2008;9:162-76.

6. Mayinger P. Phosphoinositides and vesicular membrane traffic. Biochim Biophys Acta - Mol Cell Biol Lipids 2012;1821:1104-13.

7. Rice WL, Kaplan DL, Georgakoudi I. Two-photon microscopy for non-invasive, quantitative monitoring of stem cell differentiation. PLoS One 2010;5:e10075.

8. Rizzatti V, Boschi F, Pedrotti M, Zoico E, Sbarbati A, Zamboni M. Lipid droplets characterization in adipocyte differentiated 3T3-L1 cells: size and optical density distribution. Eur J
Histochem 2013;57:e24.

9. Gluchowski NL, Becuwe M, Walther TC, Farese RV. Lipid droplets and liver disease: from basic biology to clinical implications. Nat Rev Gastroenterol Hepatol 2017;14:343-55.

10. Papackova Z, Cahova M. Fatty acid signaling: the new function of intracellular lipases. Int J Mol Sci 2015;16:3831-55.

11. Barbosa AD, Savage DB, Siniossoglou S. Lipid droplet-organelle interactions: emerging roles in lipid metabolism. Curr Opin Cell Biol 2015;35:91-7.

12. Dennis EA. Liberating chiral lipid mediators, inflammatory enzymes, and LIPID MAPS from biological grease. J Biol Chem 2016;291:24431-48.

13. Busato A, Fumene Feruglio P, Parnigotto PP, Marzola P, Sbarbati A. In vivo imaging techniques: a new era for histochemical analysis. Eur J Histochem 2016;60:2725.

14. McCormack L, Dutkowski P, El-Badry AM, Clavien P-A. Liver transplantation using fatty livers: Always feasible? J Hepatol 2011;54:1055-62.

15. Neuschwander-Tetri BA. Non-alcoholic fatty liver disease. BMC Med 2017; $15: 45$.

16. Bellentani S. The epidemiology of nonalcoholic fatty liver disease. Liver Int 2017;37(Suppl 1):81-4.

17. Byrne CD, Targher G. NAFLD: a multisystem disease. J Hepatol 2015;62: S47-64.

18. Kucera O, Cervinkova Z. Experimental models of non-alcoholic fatty liver disease in rats. World $\mathrm{J}$ Gastroenterol 2014;20:8364-76.

19. Croce AC, Ferrigno A, Di Pasqua LG, Berardo C, Piccolini VM, Bertone V, et al. Autofluorescence discrimination of metabolic fingerprint in nutritional and genetic fatty liver models. J Photochem Photobiol B 2016;164:13-20.

20. Croce AC, Bottiroli G. Autofluorescence spectroscopy and imaging: a tool for biomedical research and diagnosis. Eur J Histochem 2014; 58:2461.

21. Fukumori T, Ohkohchi N, Tsukamoto S, Satomi S. The mechanism of injury in a steatotic liver graft during cold preservation. Transplantation 1999;67:195200.

22. Croce AC, De Simone U, Vairetti M, Ferrigno A, Boncompagni E, Freitas I, et al. Liver autofluorescence properties in animal model under altered nutritional conditions. Photochem Photobiol Sci 2008;7:1046-53.

23. Croce AC, Ferrigno A, Bertone V, Piccolini VM, Berardo C, Di Pasqua LG, et al. Fatty liver oxidative events monitored by autofluorescence optical diagnosis: a comparison between subnormothermic machine perfusion and conventional cold storage preservation. Hepatol Res 201647(7):668-82 doi:10.1111/ hepr.12779.

24. Croce AC, Ferrigno A, Vairetti M, Bertone R, Freitas I, Bottiroli G. Autofluorescence properties of isolated rat hepatocytes under different metabolic conditions. Photochem Photobiol Sci 2004;3:920-6.

25. Croce AC, Ferrigno A, Di Pasqua LG, Berardo C, Mannucci B, Bottiroli G, et al. Fluorescing fatty acids in rat fatty liver models. J Biophotonics 2016. doi: 10.1002/jbio. 201600195

26. Calder PC. Fatty acids and inflammation: The cutting edge between food and pharma. Eur J Pharmacol 2011;668: S50-8.

27. Bennett M, Gilroy DW. Lipid mediators in inflammation. Microbiol Spectr 2016;4. doi:10.1128/microbiolspec. MCHD-0035-2016.

28. Yokoyama Y, Nimura Y, Nagino M, Bland KI, Chaudry IH. Role of thromboxane in producing hepatic injury during hepatic stress. Arch Surg 2005;140: 801-7.

29. Tam VC. Lipidomic profiling of bioactive lipids by mass spectrometry during microbial infections. Semin Immunol 2013;25:240-8.

30. Katagiri H, Ito Y, Ishii K, Hayashi I, Suematsu M, Yamashina S, et al. Role of thromboxane derived from COX-1 and -2 in hepatic microcirculatory dysfunction during endotoxemia in mice. Hepatology 2004;39:139-50.

31. Vairetti M, Ferrigno A, Rizzo V, Richelmi P, Boncompagni E, Neri D, et al. Subnormothermic machine perfusion protects against rat liver preservation injury: A comparative evaluation with conventional cold storage. Transplant Proc 2007;39:1765-7.

32. Ferrigno A, Carlucci F, Tabucchi A, Tommassini V, Rizzo V, Richelmi P, et al. Different susceptibility of liver grafts from lean and obese Zucker rats to preservation injury. Cryobiology 2009;59:327-34.

33. Boncompagni E, Gini E, Ferrigno A, Milanesi G, Gringeri E, Barni S, et al. Decreased apoptosis in fatty livers submitted to subnormothermic machineperfusion respect to cold storage. Eur J Histochem 2011;55:e40.

34. Malarkey DE, Johnson K, Ryan L, Boorman G, Maronpot RR. New Insights into Functional Aspects of Liver Morphology. Toxicol Pathol 
37. Hall Z, Bond NJ, Ashmore T, Sanders F, Ament Z, Wang X, et al. Lipid zonation and phospholipid remodeling in nonalcoholic fatty liver disease. Hepatology 2017;65:1165-80. .

38. Loomba R, Quehenberger O, Armando A, Dennis EA. Polyunsaturated fatty acid metabolites as novel lipidomic biomarkers for noninvasive diagnosis of nonalcoholic steatohepatitis. J Lipid Res 2015;56:185-92.
39. Croce AC, Di Pasqua LG, Berardo C, SicilianoV. Ferrigno A, Bottiroli G, Vairetti MP. Real time optical liquid biopsy: serum fatty acid profiling in a rat model of nonalcoholic steatohepatitis (NASH). Lasers Surg Med 2017;49:E5-418.

40. El-Badry AM, Graf R, Clavien P-A. Omega 3 - Omega 6: What is right for the liver? J Hepatol 2007;47:718-25. 